\title{
Presentation in Microscopy: Selection of Color to Accommodate Those with Color Vision Deficiency
}

\author{
Douglas R. Keene ${ }^{1}$ and Sara F. Tufa ${ }^{1}$ \\ ${ }^{1}$ MicroImaging Center, Shriners Hospitals for Children, Portland, Oregon
}

With the prevalence of tissue stains and fluorophores used as visualization tools in the life sciences and specifically in microscopy, consideration of the viewer's ability to discriminate color variation becomes relevant. This abstract is presented so that those within the microscopy community might be aware of the prevalence of color vision deficiency and to suggest easily accessed tools which allow a microscopist with normal color perception to view images as if they were affected, and to substitute suitable colors accordingly.

Color blindness, or color vision deficiency, is an inherited condition known to affect up to $8 \%$ of the male and $0.4 \%$ of the female population of Northern European ancestry. The most common forms, protonopia and deuteranopia, are X-linked conditions that affect the ability to perceive the colors red and green due to an absence of the associated photoreceptors [1], [2].

Color blind himself, John Dalton, who is best known for publishing atomic theory and the first table of relative atomic weights (six elements) also communicated the first formal description regarding color blindness. Besides blue and purple he was able to recognize only one color of the spectrum, yellow. Within his 1794 paper: "Extraordinary Facts Relating to the Vision of Colours" was his testimony: "That part of the image which others call red appears to me little more than a shade or defect of light. After that the orange, yellow and green seem one colour which descends pretty uniformly from an intense to a rare yellow, making what I should call different shades of yellow" [3]. He postulated that the shortage in color perception was caused by discoloration of the liquid medium of the eyeball, which has since been proven incorrect. Examination of his own preserved eyeball in 1995 demonstrated that Dalton had deuteroanopia, in which medium wavelength sensitive cones are missing. Another testimony from the modern web: "I have acute RG (Red/Green) color blindness. I can differentiate blue/cyan, $\mathrm{red} / \mathrm{magenta}$ all from black, and blue/cyan from red/magenta. Blue is good, but yellow only works if there is a background wash. In many cases, yellow is too thin on a white background."

A particularly valuable tool, available within most microscopy facilities, is a component of Adobe Photoshop versions CS4 and later. This tool allows one to open a color image, then to convert it to the interpretation perceived by an individual affected with either protonopia or deuteranopia. The image pairs shown in Figures 1, 2 and 3 were composed using this tool via the following sequence: Open the image in Adobe Photoshop/View/Proof Setup/Color Blindness-Deuteranopia.

\section{References:}

[1] B. Wong. Nature Methods 8, (2011) p. 441.

[2] S. Deeb. Clinical Genetics, 64 (2005) p. 369-377.

[3] J. Dalton. Extraordinary Facts Relating to the Vision of Colors, with Observations. Memoirs of the Literary and Philosophical Society of Manchester 5 (1798) p. 28-45.

[4] This work is supported by the Shriners Hospitals for Children. 

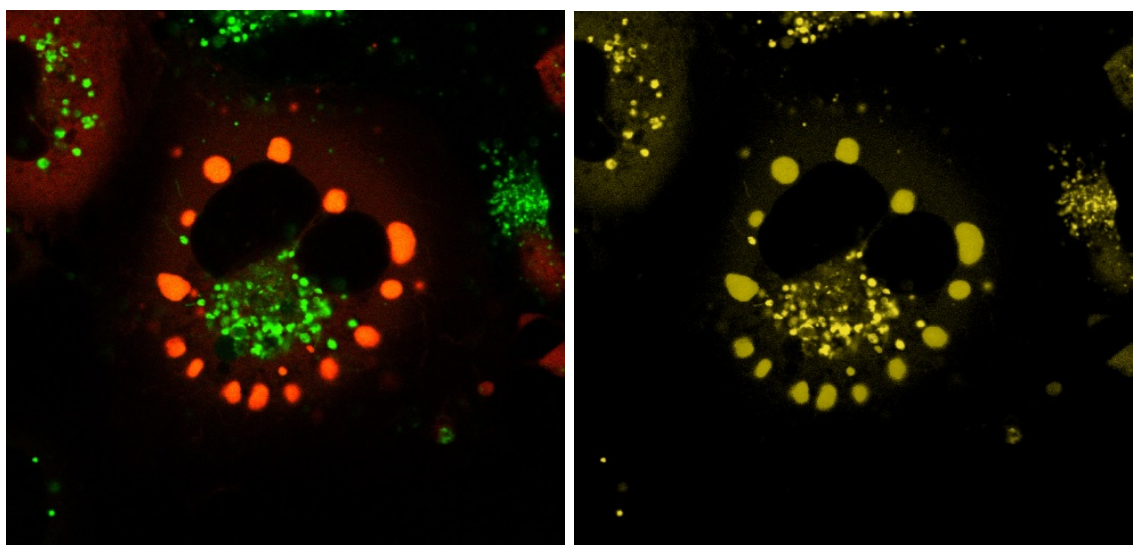

Figure 1. Two-color images from various imaging modalities are often colored red and green, as is the image on the left collected via laser scanning confocal microscopy. Converted in Photoshop, an individual with deuteroanopia perceive red and green as shades of yellow.
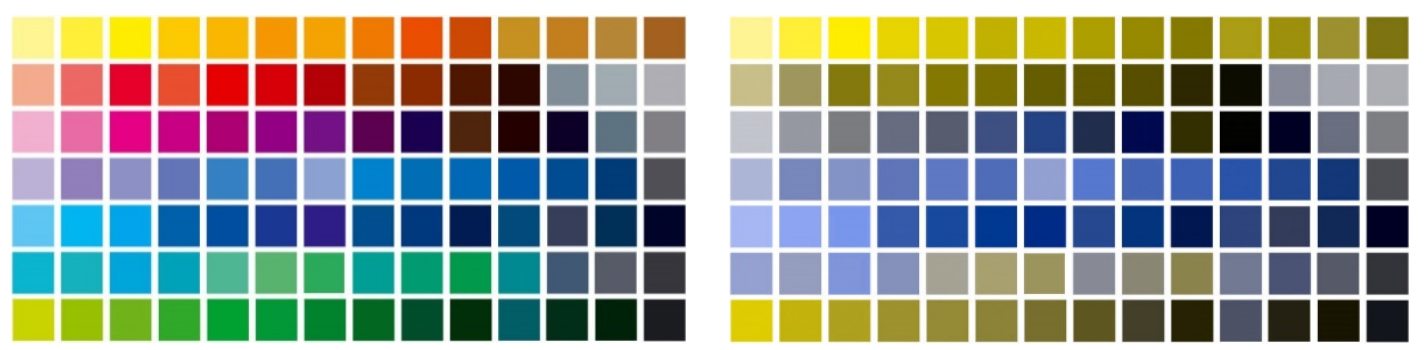

Figure 2. The color spectrum seen by an unaffected individual is shown on the left. The image on the right is the result of conversion in Photoshop to the spectrum perceived by an individual with deuteroanopia. Note that the only distinguishing colors are shades of yellow and blue.
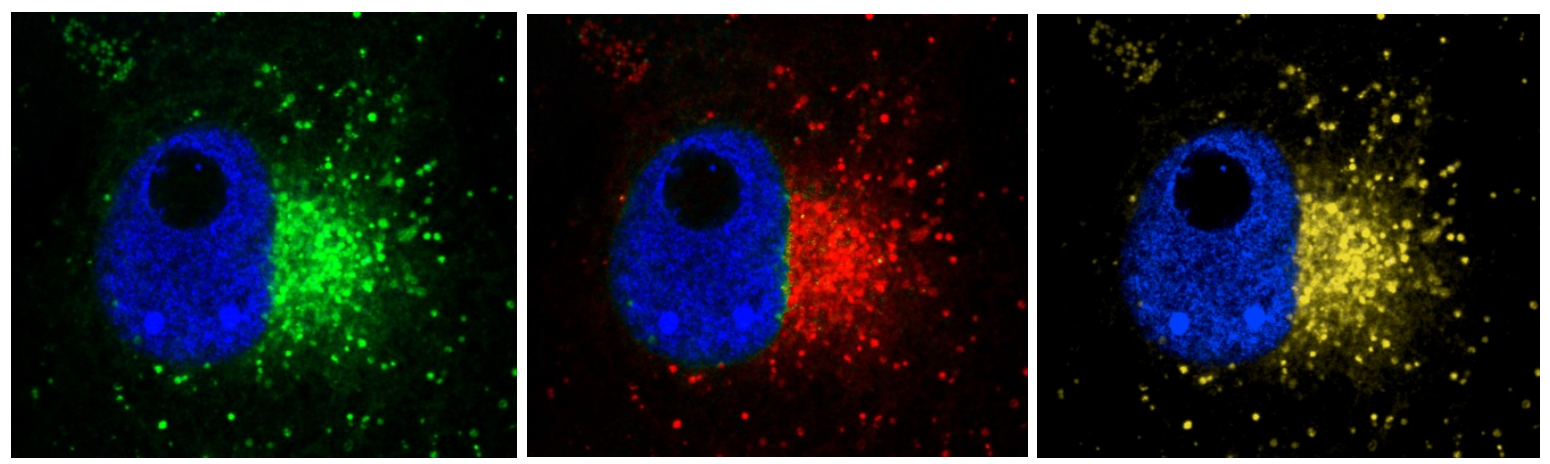

Figure 3. The regions distinguished by color variation in the left and middle images are also distinguished by individuals with deuteropia; however the color interpretation for both blue/greeen and blue/red is blue/yellow (right). To adjust color images, individual colors can be manipulated by selecting the color to be changed in Adobe Photoshop (Image/Adjustments/Hue/). 